\title{
Empire, croisades, humanités numériques : autour de l'International Medieval Congress 2014 de Leeds
}

\section{Florian Besson et Catherine Kikuchi}

\section{Q OpenEdition}

Édition électronique

URL : https://journals.openedition.org/cem/13507

DOI : $10.4000 /$ cem. 13507

ISSN : 1954-3093

Éditeur

Centre d'études médiévales Saint-Germain d'Auxerre

Référence électronique

Florian Besson et Catherine Kikuchi, « Empire, croisades, humanités numériques : autour de I'International Medieval Congress 2014 de Leeds », Bulletin du centre d'études médiévales d'Auxerre I BUCEMA [En ligne], 18.2 | 2014, mis en ligne le 19 décembre 2014, consulté le 22 septembre 2022. URL : http://journals.openedition.org/cem/13507 ; DOI : https://doi.org/10.4000/cem.13507

Ce document a été généré automatiquement le 22 septembre 2022.

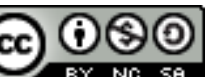

Creative Commons - Attribution - Pas d'Utilisation Commerciale - Partage dans les Mêmes Conditions 4.0 International - CC BY-NC-SA 4.0

https://creativecommons.org/licenses/by-nc-sa/4.0/ 


\title{
Empire, croisades, humanités numériques : autour de l'International Medieval Congress 2014 de Leeds
}

\author{
Florian Besson et Catherine Kikuchi
}

Du 7 au 10 juillet 2014 a eu lieu à Leeds le désormais classique et célèbre International Medieval Congress [IMC]. Se tenant désormais sur le campus de l'université de Leeds (Royaume-Uni), l'IMC a accueilli cette année plus de deux mille médiévistes, issus d'une cinquantaine de pays. Pendant les quatre jours, ont été organisées 545 sessions, tables rondes, discussions. Nous proposons ici un bref retour, forcément non exhaustif, sur ces rencontres d'importance, qui reflètent les tendances actuelles de l'historiographie, tout en contribuant à les forger et à les orienter. Pour ce faire, nous nous basons sur les

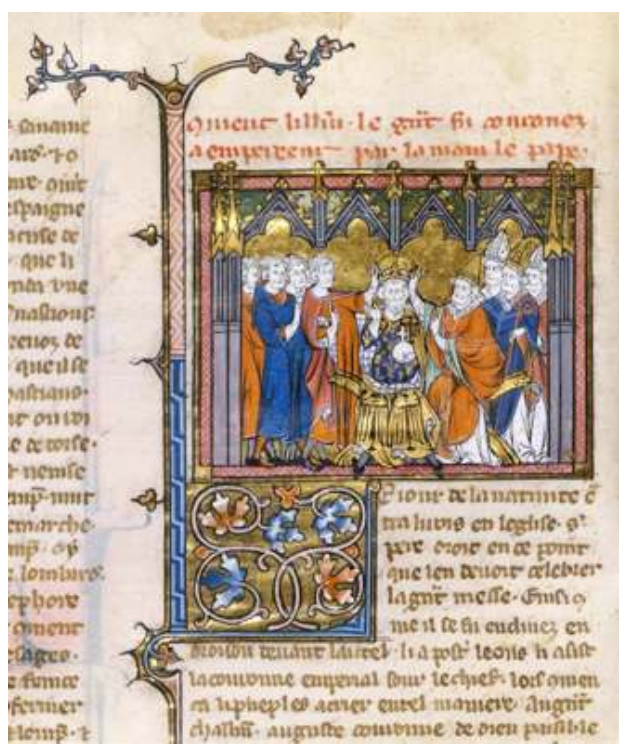
sessions auxquelles nous avons personnellement assisté, tout en n'excluant pas des considérations plus générales sur les tendances se dégageant du programme distribué par les organisateurs ${ }^{1}$. 


\section{Le thème de l'année : Empire}

2 Cette année, le thème proposé était "Empire ». Une petite moitié des sessions (204) portaient sur ce sujet, ce qui est sensiblement plus que les années précédentes et souligne à quel point le phénomène impérial attire et fascine. Porté par les New Imperial Studies $^{2}$, la recherche sur le concept d'empire est revenue au cœur de l'historiographe actuelle, en particulier, bien sûr, des recherches en histoire moderne et contemporaine, mais aussi, comme l'atteste l'IMC, en histoire médiévale. Très axé sur la pensée politique - 234 entrées dans l'index du programme des organisateurs, le congrès ne négligeait pas non plus les dimensions plus concrètes, en s'intéressant aux pouvoirs et à la diplomatie (300 entrées). L'histoire militaire ou économique, en revanche a été en retrait - respectivement 90 et 98 entrées. On peut relever les principales caractéristiques de ces sessions.

3 Tout d'abord, de très nombreuses sessions interrogeaient le sens même du concept impérial. Peut-on parler d'empire au Moyen Âge ? Y a-t-il des empires sans empereurs ? Suffit-il qu'un pouvoir politique utilise le terme d'imperium pour qu'il soit qualifié d'empire ? Ces questions ont structuré l'ensemble du congrès. Les perspectives étaient bien souvent critiques, par exemple à l'égard de «l'empire vénitien»: Georg Christ, dans son intervention "The Venetian Empire: A Historiographical Construct?", a ainsi souligné que le succès de Venise tient justement au fait de ne pas se présenter comme un empire, concept qui ne lui a été associé que bien plus tard par l'historiographie. Parmi les champs d'investigation les plus porteurs, soulignons l'importance de «l'empire Plantagenêt ». Les discussions ont souvent été animées, certains soulignant que le concept ne convient guère, en insistant notamment sur le fait que le roi d'Angleterre est le vassal du roi de France, alors qu'un empereur ne reconnait personne au-dessus de lui, voire même en dehors de lui. D'autres au contraire, entraînés en particulier par David Bates ${ }^{3}$, soutenaient que les ambitions impériales structuraient le régime angevin : la victoire d'Arthur sur les Romains peut être vue comme un symbole de la translatio imperii. Ce qui est en jeu à travers ces discussions, c'est le sens et l'utilité même du concept impérial. Ces débats ne sont pas stériles : ils poussent au contraire à conceptualiser, à généraliser, à sortir de la monographie pour proposer une analyse de fond de la structure et des dynamiques du pouvoir. Parmi les très nombreuses sessions, soulignons celle organisée par le Laboratoire de médiévistique occidentale de Paris (LAMOP) : intitulée "Marginal Empires? Imperial Practices and Representations on the Borders of Europe ». Elle a permis à Arnaud Lestremau, Olivier Viron et Fanny Madeline de s'interroger sur la place du terme imperium dans les discours et les pratiques des derniers rois anglo-saxons ou de l'Irlande des $\mathrm{X}^{\mathrm{e}}$-XI ${ }^{\mathrm{e}}$ siècles. Les discussions ont mis en avant le fait que l'empire est avant tout un rêve, polarisant l'imaginaire politique et reflétant la profonde fascination de l'Europe médiévale pour l'empire romain ${ }^{4}$.

Deuxième point fort de ces recherches sur l'empire, l'attention portée aux aires extraeuropéennes. L'Islam en particulier se taille la place du lion, du califat omeyyade à l'empire ottoman en passant par les sultans du Deccan et les émirs turcs. On relèvera en particulier la conférence (keynote lecture) de Hugh Kennedy portant sur « The End of Islamic Late Antiquity: Change and Decay in the 10th Century Middle East». Il a souhaité mettre l'accent sur la continuité qui existe entre les périodes pré- et post-islamiques pour déplacer la véritable rupture sur le plan institutionnel, politique et social au $\mathrm{x}^{\mathrm{e}}$ siècle, sous le califat abbasside. Au-delà de l'Islam, des sessions se sont interrogées 
sur l'empire en Chine - ainsi la conférence de Naomi Standen, «A Forgotten Eurasian Empire: the Liao Dynasty, 907-1125 »-, en Perse ou en Arabie préislamiques ${ }^{5}$. Des tables rondes étaient organisées dans une perspective comparatiste, mettant en présence des spécialistes de plusieurs époques et de plusieurs régions ${ }^{6}$. On remarquera l'absence quasi-totale du Japon : le shogunat n'est visiblement plus aussi «à la mode » qu'il y a quelques années, l'attention se focalisant davantage sur les mondes eurasiatiques.

De nombreuses sessions reflètent également un regain d'intérêt pour l'histoire du Saint-Empire romain. Il est significatif à cet égard que l'IMC se soit ouvert, dans une conférence, sur la Kaiserchronik, en cours d'édition critique par Mark Chinca et Christopher Young. Plusieurs intervenants ont souligné qu'il s'agissait une construction politique ambitieuse et efficace, apte à construire un discours politique complexe ${ }^{7}$. Des sessions se sont focalisées sur ses marges et ses bordures, pour explorer les rapports entre le centre et les périphéries qui apparaissent souvent comme l'un des éléments constitutifs du fait impérial ${ }^{8}$.

6 Un assez grand nombre de sessions ont souligné l'attention continue pour une vision « au féminin » de l'empire, autour de deux aspects. Tout d'abord, une vision renouvelée du queenship ${ }^{9}$ : pour beaucoup d'intervenants, il s'agissait de comprendre la manière dont les impératrices et les reines se mouvaient dans les structures politiques des empires, le rôle qui leur était octroyé ou qu'elles s'attribuaient ${ }^{10}$. D'autre part, certains ont cherché à étudier le rôle des femmes dans ces empires, que ce soit à travers leur participation politique ou dans les représentations artistiques ${ }^{11}$; on a aussi pu s'intéresser au rôle des femmes au sein de l'Empire britannique du XIX ${ }^{e}$ siècle, sous l'angle du médiévalisme et des représentations de l'anglo-saxonisme ou de la chevalerie ${ }^{12}$.

7 Notons enfin que l'Église occupe toujours une place clé. Avec près de 250 entrées, le thème "ecclesiastical history" domine l'index. De très nombreuses sessions se sont penchées sur la façon dont l'Église pense et définit le fait impérial, à travers des traités politiques ou des ouvrages théologiques. Mais l'Église est aussi elle-même une structure politique, de plus en plus centralisée, et son pouvoir a été passé au crible du concept impérial: on a pu ainsi entendre parler d'un empire spirituel - soulignons les interventions de Johnny Jakobsen ${ }^{13}$ et de Benjamin Weber ${ }^{14}$, dans une session intitulée "A Spiritual Empire in the North». Comme l'ont noté certains intervenants, l'Église est finalement la seule structure qui résiste à l'épreuve des siècles, qui pense et tente d'organiser un pouvoir universel, qui organise et dirige jusqu'à la vie quotidienne de ses sujets, par la force si besoin ${ }^{15}$. Dans le Moyen Âge occidental, l'Église catholique serait-elle l'empire par excellence?

\section{Les croisades}

8 Mis à part la thématique impériale, aucun autre sujet de recherche n'était autant représenté que les croisades - près de 230 entrées dans l'index. On comptait plusieurs sessions chaque jour sur ce thème, sessions qui ont notamment porté sur l'usage de la Bible dans les sources des croisades, sur les rapports entre ethnicité et identité dans les États latins d'Orient, sur les contacts entre les Francs et les Byzantins, sur l'imaginaire de la croisade en Occident. Ces sessions étaient animées par de prestigieux spécialistes - Alan Murray, Susan Edgington, John France -, mais faisaient aussi place à des doctorants ou jeunes chercheurs - citons entre autres Simon John, Thomas Lecaque, 
Heather Crowley... L'affluence du public était considérable, ce qui souligne à quel point le thème est porteur et continue de susciter l'imagination. Parmi de nombreuses avancées, on peut relever deux tendances de fond très importantes pour l'histoire des croisades et de l'Orient latin. Tout d'abord, les Gesta Francorum de l'Anonyme normand, qui ont longtemps été la source privilégiée pour la première croisade, sont désormais sévèrement critiquées, et on leur préfère l'Historia d'Albert d'Aix, longtemps dévalorisée et ignorée, mais que l'édition critique de Susan Edgington a largement contribué à revaloriser. Ensuite, et surtout, l'histoire des États latins d'Orient connaît une inflexion décisive : le modèle de Joshua Prawer d'une société coloniale coupée des indigènes est définitivement abandonné, au profit de modèles insistant sur les échanges et les contacts. Parmi de très nombreuses interventions de qualité, on indiquera celles de Betty Binysh " "Don't know, don't care": Challenging the Stereotype of Muslims' Views of Christians in the Holy Land", de Andrew Buck "Confraternity and the Influence of "Crusader" Identity in the Latin East: the "Crusader Community of Antioch" Revisited", et de Heather Crowley «Identity and Environments, Ethnicity and Communities: Looking at Landscapes in the Crusader States ». Le simple intitule de ces communications souligne combien le point de vue est en train de se modifier : il s'agit clairement de remettre en question les stéréotypes, de revisiter les modèles. Alors qu'on pourrait croire, vu l'ampleur de la bibliographie, que tout a été dit sur les croisades, l'IMC rappelle avec force que beaucoup reste à faire.

\section{Projets et méthodes}

9 Au-delà de ces grands thèmes, les sessions de l'IMC ont aussi permis de présenter au public de nouvelles réflexions et de nouvelles méthodes. Reflétant l'actualité de la recherche, de nombreuses sessions traitaient ainsi de l'espace, de sa production et de son organisation. On a parlé des villes et de l'urbanisation ${ }^{16}$, des espaces religieux ${ }^{17}$, des littoraux ${ }^{18}$. Une table ronde spéciale était organisée autour du thème de l'espace dans les textes médiévaux ${ }^{19}$ : celle-ci était centrée sur la manière dont les Digital Studies permettent de mieux comprendre les implications spatiales des textes médiévaux, avec un exemple analysé et discuté successivement par les différents participants. Toutes ces recherches soulignent l'importance du spatial turn, qui a véritablement inscrit l'espace et le territoire au cœur des préoccupations des historiens. Elles soulignent également la nécessité d'accompagner des nouveaux questionnements par de nouvelles méthodes d'analyse, parmi lesquelles les Digital Humanities occupent une place centrale.

10 Il est assez surprenant que les Digital Humanities n'aient fait l'objet que de peu de sessions en tant que telles, cette absence s'expliquant sans doute par la tenue des Digital Humanities Conferences de Lausanne dans le même temps. Cependant, les méthodes s'appuyant sur cet ensemble de pratiques ont fait l'objet de présentations variées. Les sessions organisées par la revue Medieval Prosopography ont ainsi permis la présentation de plusieurs recherches utilisant la prosopographie dans des contextes divers. Soulignons en particulier la présentation de Miriam Shadis, "Charting Queenship and Community in 13th century Portugal»: celle-ci a montré que la prosopographie permet d'éclairer les rôles attribués à la reine au Portugal entre 1100 et 1250, ce titre désignant aussi bien la femme du roi que ses filles, qui agissent et se font représenter comme des reines à part entière. Les deux sessions « Medieval Literacy in the Former Low Countries and in Northern France » ont également permis d'aborder différentes manières d'étudier la 
lecture et l'écriture dans les sociétés de la fin du Moyen Âge. Hélène Haug a ainsi exposé la méthode qu'elle a suivie dans sa thèse afin d'étudier la fonction et la pratique de la lecture chez les nobles de France et de Bourgogne, à travers l'utilisation d'une base de données relationnelles et de l'analyse quantitatives des données recueillies dans les sources.

11 Enfin, les présentations de plusieurs projets collectifs ont suscité des discussions méthodologiques très intéressantes. Le projet People of Medieval Scotland 1093-1314 a proposé une session très suivie: Matthew Hammond, Davit Brown et Cornell Jackson ont présenté leur collaboration, en tant qu'historiens et informaticiens, pour analyser leur base de données prosopographique grâce à l'analyse de réseaux ou Social Network Analysis ${ }^{20}$. Toutes ces présentations et ces sessions ont ainsi démontré, s'il en était besoin, que les méthodes comme les questionnements historiques sont aujourd'hui en plein renouvellement.

\section{Autour de l'IMC}

Enfin, autour des sessions et des discussions étaient également organisées de nombreuses activités : visites de la ville ou des environs, ateliers de cuisine médiévale ou de calligraphie, projections de films, entre autres. Le dernier jour, une foire médiévale accompagnée de différentes animations occupait le campus - fauconnerie, démonstration de combat médiéval, pièce de théâtre -, la journée se terminant par un bal traditionnel celtique ou ceilidh. Par ailleurs, des réceptions étaient organisées durant toute la durée du congrès pour présenter des revues (Speculum) ou des ressources - comme l'International Medieval Bibliography. Des tables rondes sur la vie académique ont été particulièrement intéressantes ${ }^{21}$; souvent trop courtes pour occasionner une véritable discussion approfondie, elles ont néanmoins le mérite d'être suggestives et de forcer à des réflexions qui peuvent d'ailleurs se poursuivre au-delà du congrès. Une grande foire aux livres se tenait également durant tout le congrès, proposant des ouvrages parfois difficiles à trouver et avec des réductions souvent très importantes. Ces différentes animations contribuent à faire de ce congrès une occasion de rencontres internationales et de libre discussion, dans une ambiance très conviviale, ce qui est précieux, notamment pour des jeunes chercheurs.

13 Difficile de rendre justice en si peu de place à un congrès si dense et à de si nombreuses interventions. Occasion de rencontres, d'échanges, de débats, l'IMC nous a également semblé être un bon prisme de certaines des tendances actuelles de la recherche, comme nous avons tenté de le souligner ici. Le prochain congrès, en juillet 2015, aura pour thème Reform and Renewal : l'histoire continue...

Reçu : 10 août 2014 - Accepté : 24 novembre 2014 


\section{NOTES}

1. Le programme est disponible en ligne à cette adresse: http://www.leeds.ac.uk/ims/imc/ imc2014.html.

2. Voir K. WILSON (ed.), A New Imperial History: Culture, Identity and Modernity in Britain and the Empire, 1660-1840, Cambridge, 2004.

3. D. BATES, The Normans and Empire, Oxford, 2013.

4. Roland Scheel le soulignait également au sujet de la Scandinavie dans une intervention intitulée «Byzantium-Rome-Denmark-Iceland: Dealing with Imperial Concepts in Scandinavia ».

5. Session "Shadows of Empire, Distant Mirrors: the Case of South Arabia», avec Walther Pohl, George Hatke, Eirik Hovden et Daniel Mahoney.

6. Session "Medieval Empire in Cross-Cultural Perspective, a Round Table discussion", avec, entre autres, David Bates, Eric Hanne, Len Scales et Jonathan Shepard.

7. Par exemple, Marcello Pacifico, «The Kingdom of Peace and Justice of Frederick II ».

8. Session "Empire and its Northern Borderlands: Advantages of Being Peripherical», avec Manja Olschowski, Michael Meichsner et Mihkel Mäesalu.

9. Voir J. C. PARSONS (dir.), Medieval Queenship, New York, 1993.

10. Par exemple les sessions «Emperor and King Sigismund of Luxembourg, II : Queenship and CoRulership» avec Amalie Fößel, Daniela Dvořáková et Anna Jagošová; ou encore « New Directions and Research in Queenship Studies » avec Zita Eva Rohr et Kathryn Maude.

11. La session « Women and Empire » avec Rebecca Browett, Başak Burcu Tekın et Barbara BoloixGallardo, a notamment abordé le rôle des femmes dans l'empire seldjoukide et dans le royaume de Grenade.

12. Session "Women and Empire: 19th-Century Medievalism, Anglo-Saxonism, and Chivalry» avec Clare Broome Saunders, Debbie Bark et Kate Lister.

13. "Dominican Stormtroopers of the Papal Empire in the Northern Europe ".

14. "How to Manage a Christendom-Wide Empire? Many Questions on Papal Collectors in the Baltic Regions in the 15th Century".

15. Voir par exemple la session «Papal Imperialism and the Empire of Evil : Heresy, Witchcraft and the expansion of Papal Power ", avec John Ward, Lola Davidson et Run Hagen.

16. Session "Urbanisation and the Church », avec Alan Kissane et Ian Eckford.

17. Sessions « Negotiating Monastic Space».

18. Sessions «Landscapes/Seascapes ».

19. "Spatial Aspects in Medieval Textes and the Potential of the Digital Humanities", table ronde organisée par Frithjof Schwartz, en visioconférence avec les Digital Humanities Conferences de Lausanne, congrès tenu à Lausanne du 7 au 12 juillet 2014.

20. On peut aussi citer les sessions organisées par le projet England's Immigrants 1330-1550, auquel participent de nombreux jeunes chercheurs.

21. Mentionnons par exemple les sessions «Women, Scholarship, and Collective Action: A Round Table Discussion» organisée par Liz Herbert McAvoy ou encore «Borders and Boundaries in the University : A Round Table Discussion » organisée par Kathryn Maude et Audrey Thorstad. 
INDEX

Mots-clés : croisade, empire, humanités numériques

\section{AUTEURS}

FLORIAN BESSON

Université Paris-Sorbonne - Paris 4

\section{CATHERINE KIKUCH}

Université Paris-Sorbonne - Paris 4 